\title{
A Compartmental Epidemiological Model Applied to the Covid-19 Epidemic
}

\author{
Anne-Flore Baron ${ }^{2}$, Olivier Boulant ${ }^{1}$, Ivan Panico ${ }^{2}$, Nicolas Vayatis ${ }^{1}$ \\ ${ }^{1}$ Université Paris-Saclay, ENS Paris-Saclay, CNRS, Centre Borelli, 94235 Cachan, France \\ (olivier.boulant@ens-paris-saclay.fr) \\ 2 onepoint, 29 rue des Sablons, 75116 Paris, France \\ (i.panico@groupeonepoint.com)
}

Communicated by Gregory Randall Demo edited by Anne-Flore Baron

\begin{abstract}
The objective of this work is to provide a sophisticated but accessible compartmental epidemic model. Our algorithm is highly inspired from the compartmental model developed by Sofonea and al. in 2020. This model has been used as a reference for several working groups in France during the Covid-19 crisis. Each individual is allocated to a compartment according to her age, her current state with respect to the disease, as well as the length of time she has been in that state. The model then reproduces the mechanisms of transition from one state to another: mathematically, this translates into a system of recurrence relations. It captures how much individuals interact with one another through a parameter that estimates compliance with hygiene measures and lifestyle habits. The present work aims to make the model implementation fully transparent as well as the corresponding code available and give control to users so that they are able to test the model in total transparency. Focus has been put on reproducibility and explanation of the various parameters. The hard-coded parameters correspond to the data for the Covid-19 epidemic in France.
\end{abstract}

\section{Source Code}

The reviewed source code and documentation for this algorithm are available from the web page of this article ${ }^{1}$. Usage instructions are included in the readme.md file of the archive.

Keywords: COVID-19; prediction model; compartmental model; contact factor

\footnotetext{
${ }^{1}$ https://doi.org/10.5201/ipol.2021.323
} 


\section{Introduction}

In the context of the Covid-19 crisis, epidemics models have been in the spotlight since they have been considered to support government decisions to contain the propagation of the virus. When the first wave of contagion reached Western Europe, the model elaborated by the team of N. Ferguson at Imperial College [7] was foreseen as a realistic model to provide forecasts on the number of casualties depending on a series of parameters (characteristics of the pandemic, sociodemographic data of the population, behavior of citizens, structure of their contact network, etc.). In the early days of the contagion in France, the use of such a model was discarded by the authorities, first because of its complexity, and second, because it required the input of many parameters which were not available with sufficient accuracy at the time. Inspired from another category of epidemic models, merely known as compartmental models, some other propositions emerged in France in order to provide tools for the policy-makers while taking into account the features of the present pandemic. The most discussed propositions were those of three teams: Institut Pasteur ([5]), INSERM ([3]), Université de Montpellier - ETE team ([4], [6]). These three approaches are sophisticated variants of the well-known SIR (Susceptible-Infected-Recovered) or the SEAIR (Susceptible-Exposed-Asymptomatic-InfectedRecovered). However, to the best of our knowledge at the time of the present work, we could not find access to open source implementation of the proposed models, therefore reducing the capacity to reproduce the results. In the present work, we propose a reproducible version of an SEAIR model which follows the principles described in [6] with a finer description of compartments and including divisions with respect to age groups and duration of stay in certain compartments.

\section{A Compartmental Model with Age and Time Subdivi- sions}

\subsection{A Compact View of the Model}

The model developed in [6] proposes to consider an extension to the plain SEAIR model by adding extra compartments to better account for the propagation process of the pandemic at the national or regional level. The compartments introduced in their model are the following:

- S : Susceptible.

- J : Non critical infectious.

- Y : Critical infectious $\rightarrow$ it designates individuals whose life is threatened by the disease. They will need to be hospitalized at some point.

- W : Other critical hospitalized patients $\rightarrow$ it designates infectious individuals in critical condition who will not benefit from a long stay in ICU. They will die either after a short stay in ICU or in another ward.

- H : Long-stay ICU hospitalized $\rightarrow$ it designates infectious individuals in critical condition who are admitted to the intensive care unit for a long stay - at least one day. After their long-stay in ICU, they may either die or recover.

- $\mathrm{R}$ : Recovered immunized.

- D : Dead. 
Furthermore, all these compartments are subdivided according to age groups. Compartments J, $\mathrm{Y}, \mathrm{H}$ and $\mathrm{W}$ also include subdivisions according to the length of time the individual has remained in that state. This subdivision allows to take into account the susceptibility of different age groups to the virus, as well as the time dimension in the evolution of the disease. The model is described schematically in Figure 1.

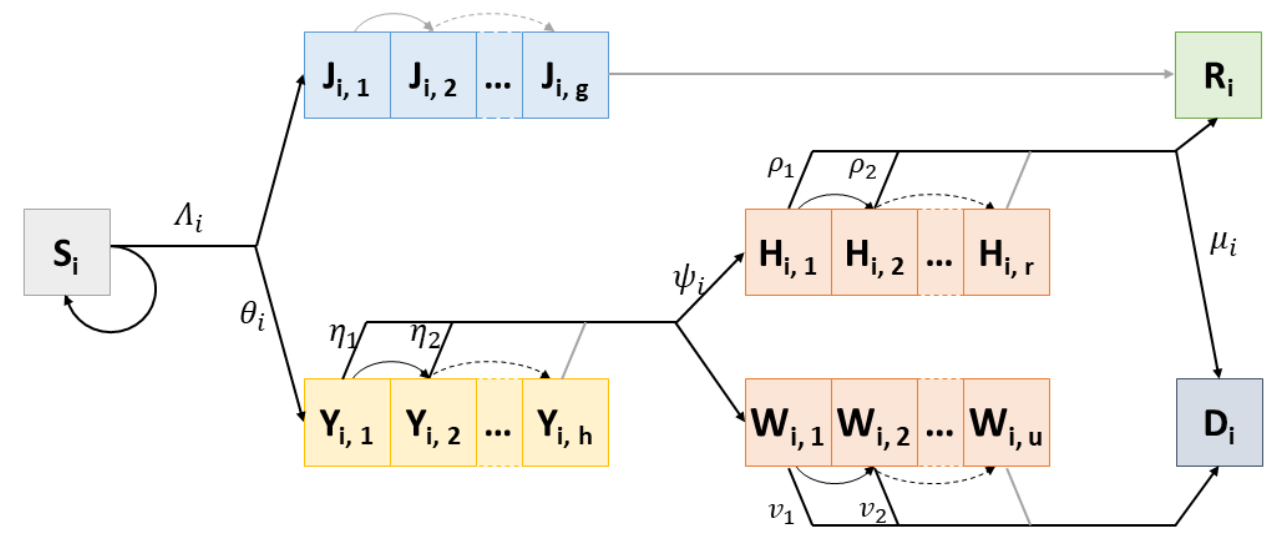

Figure 1: A compact view of the dynamics of the model. $S$ stands for susceptibles, $J$ for non critical infectious, $Y$ for critical infectious, $H$ for people in ICU, $W$ for other critical hospitalized patients, $R$ for recovered, $D$ for deaths. Each compartment is duplicated for each age group, designated by subscript $i$. Subscripts $i, j$ indicate the age group $i$ and the length of the current state $j$. Values above arrows indicate the rate of passage from the associated compartments. All these transition probabilities are fixed, except for the force of the infection $\Lambda_{i}$ calculated from the density of infected people $J+Y$ among the susceptible population $S$.

The objective of the present work is to provide a user's guide for this type of compartmentalized model, for which numerous parameters are needed. Estimation of those parameters can be complicated due to the diversity of data made available and their granularity.

\subsection{Key Parameters of the Epidemic Model}

\subsubsection{Age Groups}

The first parameters to be set are the age groups. The sub-division of the population according to their age enables us to take into account their different sensitivities to the disease and the variability of their exposure to it. Thus, the branching probabilities described below as well as the contact factor will vary according to age groups.

\subsubsection{Branching Probabilities}

These probabilities determine the ratio of individuals at a fork between two possible compartments. Their role is detailed in Figure 2 with the corresponding equations. The index $i$ of each variable relates to an arbitrary age group. For each compartment $Q, Q^{\prime}$ stands for $Q(t+1)$, the daily update of the variable $Q=Q(t)$. 


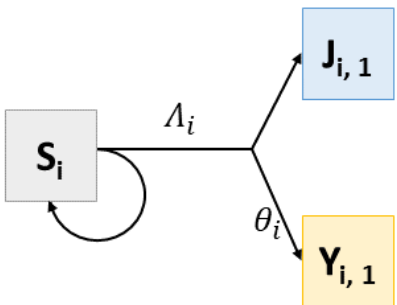

(a) $\theta_{i}$ : proportion of critical infected $Y_{i, 1}$ among the $\Lambda_{i} S_{i}$ newly infected individuals.

$J_{i, 1}^{\prime}=\left(1-\theta_{i}\right) \Lambda_{i} S_{i}$

$Y_{i, 1}^{\prime}=\theta_{i} \Lambda_{i} S_{i}$

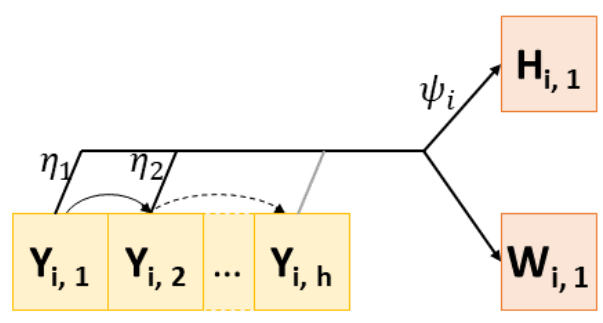

(b) $\psi_{i}$ : proportion of admissions in ICU $H_{i, 1}$ among the $\sum_{j=1}^{h} \eta_{j} Y_{i, j}$ newly hospitalized critically infected individuals.

$H_{i, 1}^{\prime}=\psi_{i} \sum_{j=1}^{h} \eta_{j} Y_{i, j}$

$W_{i, 1}^{\prime}=\left(1-\psi_{i}\right) \sum_{j=1}^{h} \eta_{j} Y_{i, j}$

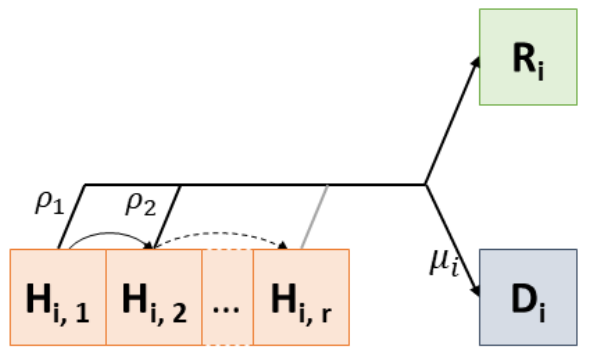

(c) $\mu_{i}$ : proportion of deaths $D_{i}$ among the $\sum_{j=1}^{r} \rho_{j} H_{i, j}$ individuals out of ICU.

$$
\begin{aligned}
& R_{i}^{\prime}=R_{i}+J_{i, g}+\left(1-\mu_{i}\right) \sum_{j=1}^{r} \rho_{j} H_{i, j} \\
& D_{i}^{\prime}=D_{i}+\sum_{j=1}^{u} v_{j} W_{i, j}+\mu_{i} \sum_{j=1}^{r} \rho_{j} H_{i, j}
\end{aligned}
$$

Figure 2: Segments of Figure 1 to illustrate the role of branching probabilities.

- $\theta_{i}$ is the ratio of critical infected $Y$ among all infected individuals $Y+J$, also called critical illness frequency. Its purpose is illustrated in Figure 2(a).

- $\psi_{i}$ is the ratio of individuals $H$ among all hospitalized individuals $H+W$ who are admitted to intensive care for more than one day. $\psi_{i}$ is also called long-stay ICU admission frequency. Its purpose is illustrated in Figure 2(b).

- $\mu_{i}$ is the ratio of dead individuals $D$ among individuals discharged from ICU $D+R$, also called long-stay ICU fatality rate. Its purpose is illustrated in Figure 2(c).

These ratios strongly depend on the age groups $i$. The age groups and these ratios (except $\mu_{i}$ ) are computed from other metrics. The proposed values can be found in Table 1.

\subsubsection{Interval Distributions}

At every day step of the simulation the states are updated. The interval distributions determine whether an individual transitions to a new state or stays in the same one by moving on to the next time sub-division within this same state. The update method is not the same for all time sub-divided states. Namely : 


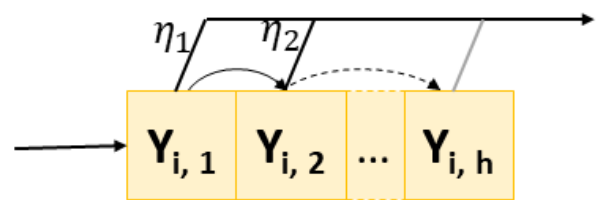

(a) $\eta_{j}$ : Probability for an individual critically infected for $j$ days to be hospitalized.

$\sum_{j=1}^{h} \eta_{j} Y_{i, j}$ individuals out of $Y$ each
day.

$Y_{i, j}^{\prime}=\left(1-\eta_{j-1}\right) Y_{i, j-1} \quad 1<j \leq h$

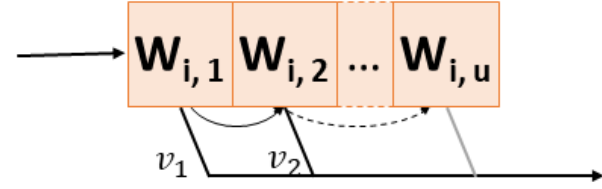

(c) $v_{j}$ : Probability for a critical individual hospitalized in a non intensive care unit for $j$ days to die. $\sum_{j=1}^{u} v_{j} W_{i, j}$ individuals out of $W$ each
day.

$W_{i, j}^{\prime}=\left(1-v_{j-1}\right) W_{i, j-1} \quad 1<j \leq u$

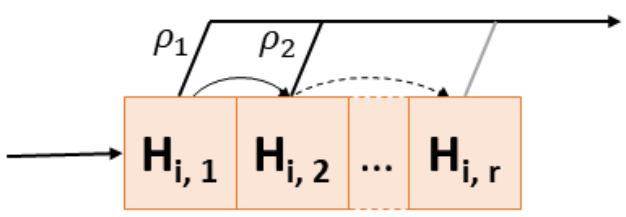

(b) $\rho_{j}$ : Probability for an individual in ICU for $j$ days to leave ICU.

$\sum_{j=1}^{r} \rho_{j} H_{i, j}$ individuals out of $H$ each
day.

$H_{i, j}^{\prime}=\left(1-\rho_{j-1}\right) H_{i, j-1} \quad 1<j \leq r$

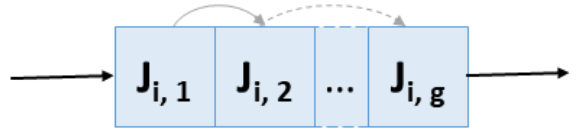

(d) Individuals in $J$ stay there for a fixed period of time $g$. They move deterministically from one time sub-compartment to the next one.

$J_{i, g}$ individuals out of $J$ each day.

$J_{i, j}^{\prime}=J_{i, j-1} \quad 1<j \leq g$

Figure 3: Segments of Figure 1 to illustrate the role of interval distributions.

- Individuals in $Y, H$ or $W$ remain in these compartments for variable durations. It is then necessary to determine for each of these compartments, a probability distribution that associates to the current time of the state, the probability of leaving it. This distribution will also determine the maximum duration of each state $h, r$ and $u$ (for $Y, H$ and $W$ respectively). The transitions of $Y, H$ and $W$ time sub-divisions are depicted respectively in Figures 3(a), 3(b) and $3(\mathrm{c})$.

- Individuals in $J$ remain in this state until they are no longer considered contagious. For the sake of simplicity, the average probability of contamination according to the time since infection has been considered: it is the generation time distribution $\zeta$. It results in a fixed duration $g$ of the $J$-state for all individuals. The transitions of $J$ sub-divisions are depicted in Figure $3(\mathrm{~d})$.

For states that do not include time sub-divisions, the update method is simpler:

- The transition probability of $S$ depends on the force of infection $\Lambda_{i}(t)$ (Definition 1$)$.

- $R$ and $D$ are stable states. Individuals in these states remain in them. This assumes that a recovered individual cannot be re-infected. This remains a daring hypothesis in view of current knowledge of the disease $([1,8])$. 
At the end, the only compartments that require interval distributions are $Y, H$ and $W$.

Definition 1. The force of infection $\Lambda_{i}(\boldsymbol{t})$ is the probability of being infected for a susceptible individual in age group $i$. This value is calculated daily from the current state of the population and the transmission parameters.

\subsubsection{Transmission Parameters}

For the computation of the force of infection $\Lambda_{i}$ mentioned above, some additional parameters are needed. The transmission parameters are provided in the following definitions.

Definition 2. The generation time distribution $\zeta_{j}$ is the distribution of the delay between the onset of the disease in one individual and its transmission to another one.

Definition 3. The basic reproduction number $\mathcal{R}_{0}$ used in this model is the number of secondary infections generated by an infected individual during her entire infectious period at the onset of the epidemic.

Definition 4. The contact factor $c_{i}(t)$ is a time-dependent behavioral parameter for each age group which estimates the density of human contacts between individuals (ranging from zero to one).

The contact factor depends mostly on the political measures and the behavior of the population. This parameter will temper the basic reproduction number $\mathcal{R}_{0}$ for the computation of the force of the infection (Equation 9). The closer the contact factor is to 0, the lower the force of infection. The aim of this parameter is to take into account, within the dynamics of the epidemic, the temporal variations in exposure to the virus of the different age groups.

We note that, in order to limit the number of parameters of the demo, the contact factor is common to all age classes, and varies on the lockdown and deconfinement dates. The contact factor can be specified more precisely in the code.

\subsection{System of Recurrence Relations}

Algorithm 1 gives an overview of how the compartments are updated during a simulation. In this section the system of recurrence relations below details the dynamics of each compartment.

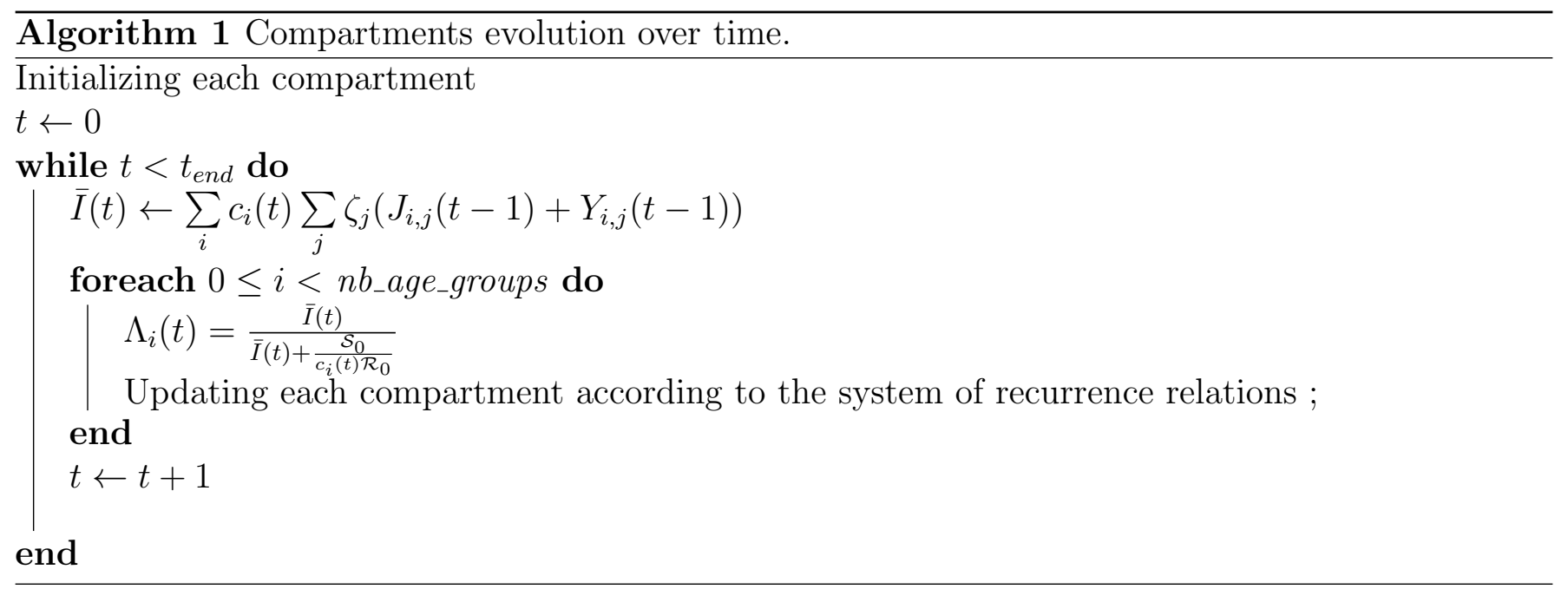

Identically to previous notations, $Q^{\prime}=Q(t+1)$ refers to the next daily value of the $Q$ compartment. Figure 1 provides a schematic view of the model for an arbitrary age group $i$, where the rate from one compartment to another is indicated above the corresponding arrow. 


$$
\begin{aligned}
S_{i}^{\prime} & =\left(1-\Lambda_{i}\right) S_{i}, & & \\
J_{i, 1}^{\prime} & =\left(1-\theta_{i}\right) \Lambda_{i} S_{i}, & J_{i, j}^{\prime}=J_{i, j-1}, & 1<j \leq g, \\
Y_{i, 1}^{\prime} & =\theta_{i} \Lambda_{i} S_{i}, & Y_{i, j}^{\prime}=\left(1-\eta_{j-1}\right) Y_{i, j-1}, & 1<j \leq h, \\
H_{i, 1}^{\prime} & =\psi_{i} \sum_{j=1}^{h} \eta_{j} Y_{i, j}, & H_{i, j}^{\prime}=\left(1-\rho_{j-1}\right) H_{i, j-1}, & 1<j \leq r, \\
W_{i, 1}^{\prime} & =\left(1-\psi_{i}\right) \sum_{j=1}^{h} \eta_{j} Y_{i, j}, & & \\
R_{i}^{\prime} & =R_{i}+J_{i, g}+\left(1-\mu_{i}\right) \sum_{j=1}^{r} \rho_{j} H_{i, j}, & W_{i, j}^{\prime}=\left(1-v_{j-1}\right) W_{i, j-1}, & \\
D_{i}^{\prime} & =D_{i}+\sum_{j=1}^{u} v_{j} W_{i, j}+\mu_{i} \sum_{j=1}^{r} \rho_{j} H_{i, j} . & &
\end{aligned}
$$

The force of infection $\Lambda_{i}$ is computed for each age group. For a susceptible individual of group $i$ exposed at date $t$, it corresponds to the daily probability of being infected. To estimate it, we begin by calculating $\bar{I}(t)$, the density of contagious individuals in the non-hospital community, weighted by the degree of social distancing of each group $c_{i}(t)$ (ranging from a simple barrier gesture to total confinement) as well as the degree of contagiousness corresponding to the time elapsed since the beginning of the infection $\zeta_{j}$ of the individuals.

$$
\bar{I}(t)=\sum_{i} c_{i}(t) \sum_{j} \zeta_{j}\left(J_{i, j}(t)+Y_{i, j}(t)\right) .
$$

The force of infection can then be deduced from this

$$
\Lambda_{i}:=\frac{\bar{I}(t)}{\frac{\mathcal{S}_{0}}{c_{i}(t) \mathcal{R}_{0}}+\bar{I}(t)} .
$$

This system of recurrence relations has an advantage over regular ODEs in the sense that it does not require resolution by integration: next day's reports can be calculated directly from the previous day.

\section{Value Recommendations for Key Parameters}

In order to run an experiment, some parameters are hard-coded or computed from others:

- age groups,

- branching probabilities.

The user can also select the following parameters:

- ICU fatality rate (used to compute some branching probabilities),

- interval distributions,

- transmission parameters,

- a set of initial conditions. 


\subsection{Age Groups}

It is crucial to differentiate between age groups that respond unequally to the disease and are targeted differently by political measures (school closure, remote work, ...). The choice of age groups should be made in such a way that:

- it is possible to differentiate groups affected by different policy measures,

- data is available to compute the branching probabilities for each group.

The ETE team [6] chose 3 age groups: $0-24,25-64$ and $65+$. Then the branching probabilities were computed by extrapolating data from other age groups. To avoid as much as possible extrapolation, we have chosen the most common age groups in the data, that is : $0-9,10-19,20-29$, $30-39,40-49,50-59,60-69,70-79,80-89,90+$.

\subsection{Branching Probabilities}

We need to find three branching probabilities:

- $\theta_{i}$ : critical illness frequency,

- $\psi_{i}$ : long-stay ICU admission frequency among critically infected individuals,

- $\mu_{i}$ : long-stay ICU fatality rate.

The long-stay ICU admission frequency among critically infected individuals $\psi_{i}$ is not a value we can directly find in public data. We compute the three branching probabilities in a similar way as [6], based on four other metrics (estimated for each age group $i$ ):

- Infection Fatality Rate (IFR): death probability among all infected individuals.

- Proportion of hospitalized patients - critical or not - admitted in ICU $u_{i}$.

- Death probability among those hospitalized (ICU or not) $d_{i}$.

- Long-stay ICU fatality rate $\mu_{i}$.

The first three metrics are estimated in [5] and hard-coded in the present work. Values can be found in Table 1. For the long-stay ICU fatality rate $\mu_{i}$, we propose by default values calculated from French public health data [2], but they can be modified in the parameters.

We can then estimate successively

$$
\begin{aligned}
\hat{\psi}_{i} & :=\frac{1}{1-\mu_{i}+\frac{d_{i}}{u_{i}}}, \\
\text { and then } \quad \hat{\theta}_{i} & :=\frac{I F R_{i}}{1-\left(1-\mu_{i}\right) \hat{\psi}_{i}} .
\end{aligned}
$$

It can be noted that the ETE team [6] calculates these parameters with similar methods and then calibrates them on the data with a correction factor. 


\begin{tabular}{lccccccccc} 
Age group & $0-19$ & $20-29$ & $30-39$ & $40-49$ & $50-59$ & $60-69$ & $70-79$ & $80+$ & Source \\
\hline Fatality rate in ICU $\mu(\%)$ & 5.6 & 4.4 & 4.4 & 6.6 & 8.6 & 12.9 & 21.8 & 32.7 & {$[2]^{*}$} \\
$p(H \mid U)(\%)$ & 22.2 & 11.6 & 15.9 & 22.2 & 27.6 & 30.8 & 24.9 & 5.6 & {$[5]$} \\
$p(D \mid U)(\%)$ & 0.6 & 1.1 & 1.9 & 3.3 & 6.5 & 12.6 & 21.0 & 31.6 & {$[5]$} \\
Infection Fatality Rate $(\%)$ & 0.001 & 0.005 & 0.02 & 0.05 & 0.2 & 0.7 & 1.9 & 8.3 & {$[5]$}
\end{tabular}

Table 1: Chosen branching parameters. $U$ corresponds to all infected hospitalized individuals - critically infected or not. (*Adapted age groups.)

\subsection{Interval Distributions}

In Section 2.2, we came to the conclusion that only $Y, H$ and $W$ needed a transition policy. We implemented the distributions suggested by [6].

- Choosing a distribution for the time spent in the state:

- Weibull distribution, with shape parameters greater than one for the duration of $Y$. $F_{w}\left(x, k, \lambda_{w}\right)=1-e^{-\left(x / \lambda_{w}\right)^{k}}$

- Exponential distributions for the durations of $H$ and $W$. $F_{e}\left(x, \lambda_{e}\right)=1-e^{-\lambda_{e} x}$

We note $F$ the chosen cumulative distribution function.

- Truncating the distribution so as to have a finite number of time sub-divisions. The truncation is carried out at the upper-integer-rounded 99\%-quantile of the original distribution. Then we have to normalize the new cumulative distribution function obtained.

$$
F^{*}(x)=\frac{F(x)}{F\left(x_{m}\right)} \forall x \in\left[0, x_{m}\right] \text { where } x_{m}=\underset{x \in \mathbf{N}}{\operatorname{argmin}}\{F(x) \geq 0.99\} .
$$

- Computing the probability of exiting the state at day $j$, conditioned by not having exited the state previously.

$$
p_{j}=\frac{F^{*}(j)-F^{*}(j-1)}{1-F^{*}(j-1)} \text { for } 1 \leq j \leq x_{m}
$$

The choice of the parameters $\left(\lambda_{w}, j\right)$ of the Weibull distribution for the duration of $Y$ and the parameter $\lambda_{e}$ for the exponential law for the durations of $H$ and $W$ are left to the user's choice. However, we draw the reader's attention to the role of $\lambda_{w}$ and $\lambda_{e}$, which varies from one distribution to another as can be seen in the cumulative distribution functions. A Weibull distribution with parameters $\left(\lambda_{w}, j=1\right)$ is equivalent to an exponential law with parameter $\lambda_{e}=\frac{1}{\lambda_{w}}$. All these probabilities are computed in the function parameters_init().

The default parameters of the interval distributions can be modified in the json parameter file epidemiological_parameters.json:

- Contamination to hospitalization interval distribution - k: 3.6

- Contamination to hospitalization interval distribution - $\lambda_{w}: 16.1$

- Long stay ICU interval distribution - $\lambda_{e}$ : 0.06

- Other critical hospitalized patients interval distribution $-\lambda_{e}: 0.15$ 


\subsection{Transmission Parameters}

Three parameters must be determined to compute the force of infection $\Lambda$ :

- the basic reproduction number $\mathcal{R}_{0}$,

- the contact factor $c_{i}$,

- the generation time distribution $\zeta_{i}$.

The basic reproduction number $\mathcal{R}_{0}$ and the contact factor $c_{i}$ are chosen by the user. The contact factor varies over the course of the epidemic. It can be interesting to specify it from one age group to another, for example to model school closures. For reasons of simplicity, the parameters available in the demo only allow the contact factor to vary over time. The generation time distribution is computed with the same method as for the interval distribution, using a Weibull distribution with chosen parameters. The only difference is that the probability does not need to be conditioned, since transmitting the virus on day $j$ is not considered to have any impact on the probability of transmission in the following days. $F$ being the cumulative distribution function of the chosen distribution as in the previous sub-section, we obtain

$$
\begin{array}{r}
F^{*}(x)=\frac{F(x)}{F(g)} \forall x \in[0, g] \text { where } g=\underset{x \in \mathbf{N}}{\operatorname{argmin}}\{F(x) \geq 0.99\}, \\
\zeta_{j}=F^{*}(j)-F^{*}(j-1) \text { for } 1 \leq j \leq g,
\end{array}
$$

where $g$ determines the number of time sub-sections in $J$. As we can see in Figure $3(\mathrm{~d})$, all non critical infected individuals stay in $J$ for $g$ days.

The default transmission parameters can be modified in the epidemiological_parameters.json file:

- Generation time distribution - $k: 2.24$

- Generation time distribution - $\lambda_{w}: 5.42$

The contact factor can be defined over time in the user interface, along with the basic reproduction number.

\subsection{Initial Conditions}

Initial conditions should be chosen depending on the country/region. More precisely, we let the user choose the total number of individuals in the population $N^{0}$. Please note that nursing homes are not included in this model and then the 730,000 French people in nursing homes should not be included in this initialization. The default values proposed in the user interface correspond to the French population as on January 1, 2020 according to INSEE data.

In our code, initialization is done in the function conditions_category_init() in model/init_utils.py. It initializes the simulation at the very beginning of the epidemic based on the following rules:

- $J$ is initialized with $J_{i, 0}=1 / n b_{-}$age_groups in each age group $i$ in the first temporal subcompartment. All other temporal sub-compartments are initialized to zero.

- $Y, H, W, R$ and $D$ are initialized to zero.

- $S$ is deduced from the total number of studied individuals: $S_{i}(0)=N_{0, i}-\frac{1}{\text { nb_age_groups }}$ for each age group $i$. 
However, to initialize the model in the middle of an outbreak, other methods can be considered and implemented instead of the function conditions_category_init():

- Individuals can be distributed equally across all time compartments.

- One can find the geometric sequence with common ratio $\mathcal{R}_{0} c_{i}(0)$ so as to have the right number of people in total.

As the distribution of individuals within the compartments at initialisation has a great impact on the results, it is important to think carefully about the selected approach.

\section{A Simulation Example under the Demo Mode}

In this section, we present the graphical outputs produced by a simulation run. In order to do so, we have to set the parameters introduced by the model and presented above.

\subsection{Example Parameters}

- Transmission parameters

- Basic reproduction number: 2.46

- Scenario parameters

- Starting day of the simulation: 2020-01-03

- Simulation duration: 350

- Contact factor evolution: In the results below the contact factor is updated three times:

- 2020-03-17: 0.5

- 2020-05-29: 0.7

- 2020-10-31: 0.5

In the demo, the contact factor can only be updated three times for reasons of simplicity. These parameters correspond approximately to 0.5 during lockdowns or curfews, and 0.7 for barrier gestures and mask use. It is a very simplified version of the French evolution during the year 2020 .

- Total number of individuals $N^{0}$ at time 0 : Table 2

\begin{tabular}{lccccccccccc} 
Age group & $0-9$ & $10-19$ & $20-29$ & $30-39$ & $40-49$ & $50-59$ & $60-69$ & $70-79$ & $80-89$ & $90+$ & Source \\
\hline$N^{0}\left(10^{6}\right)$ & 7.76 & 8.33 & 7.47 & 8.29 & 8.59 & 8.79 & 8.00 & 5.70 & 2.86 & 0.56 & INSEE
\end{tabular}

Table 2: Number of individuals $N^{0}$ per age group at time 0 (in millions). 


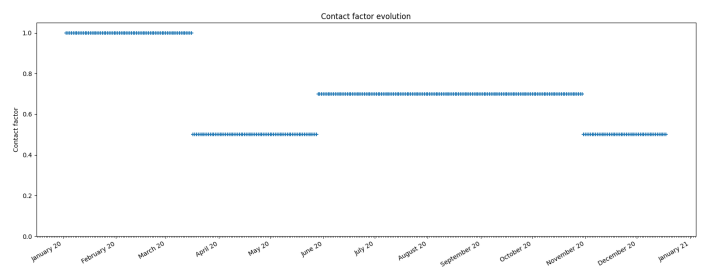

(a) Example plots of the contact factor evolution.

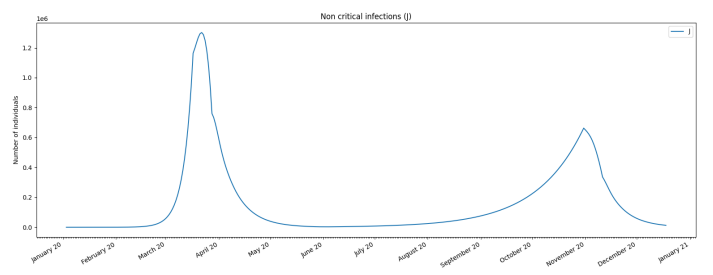

(c) Example response for number of non critical infections.

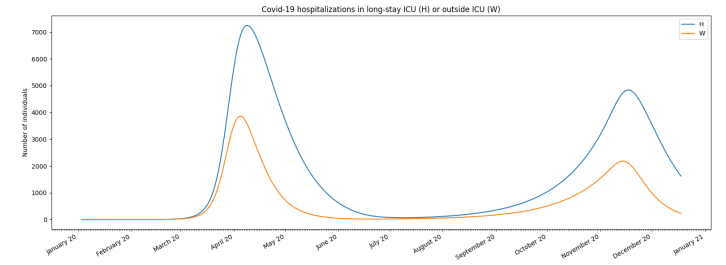

(e) Example response for number of critical individuals hospitalized or in ICU.

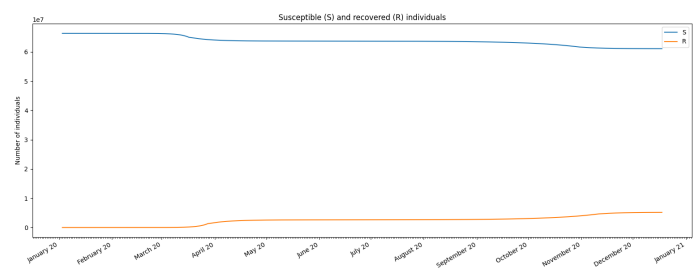

(b) Example response for number of susceptible and recovered individuals.

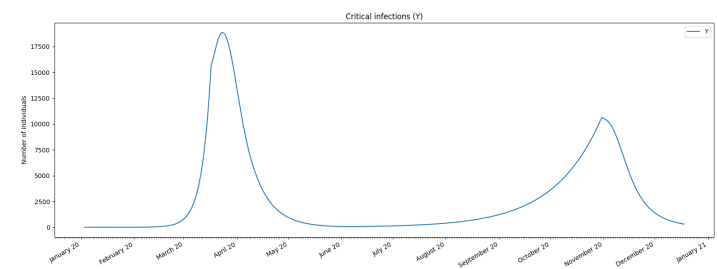

- (d) Example response for number of critical infections.

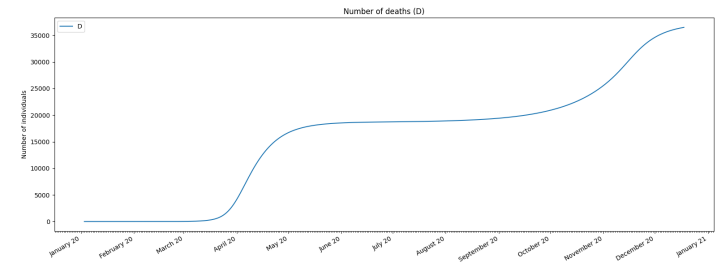

(f) Example response for number of deaths.

Figure 4: Graphical outputs of the model.

\subsection{Simulation Graphical Outputs}

All the model parameters have now been chosen. A simulation run then outputs the following figures:

- Figure 4a shows the evolution of the contact factor over time. The plot displays the instructions from the user interface.

- Figure $4 \mathrm{~b}$ shows the number of both susceptible (blue) and recovered (orange) individuals over time.

- Figure 4c shows the number of non critical infected individuals over time.

- Figure 4d shows the number of critical infected individuals over time.

- Figure $4 \mathrm{e}$ shows the number of critical infected in ICU (blue) or hospitalized outside ICU (orange) over time.

- Figure $4 \mathrm{f}$ shows the number of deaths over time. 


\section{A More Advanced Simulation Example Based on the Code Made Available}

Figure 5 shows the results that can be obtained with our code. The contact factor evolves in a much more complex way than what is allowed in the demo interface. The objective is to give a glimpse of the potential of the algorithm.

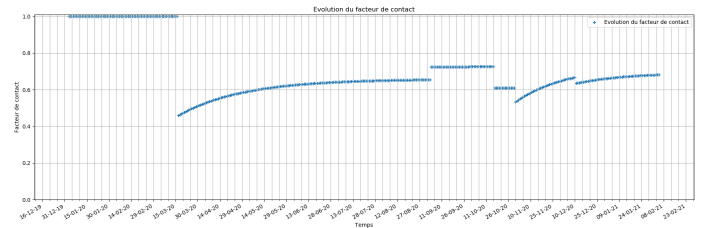

(a) Example plots of the contact factor evolution.

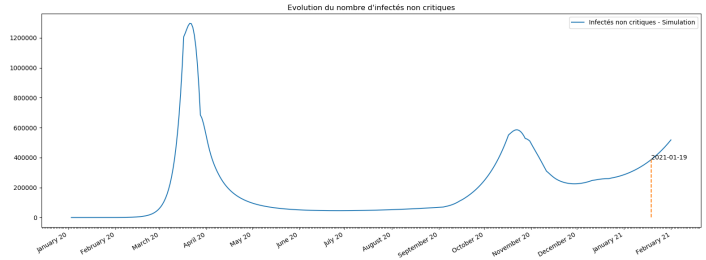

(c) Example response for number of non critical infections.

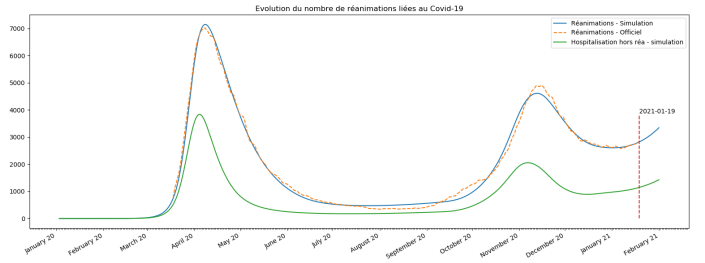

(e) Example response for number of critical individuals hospitalized or in ICU.

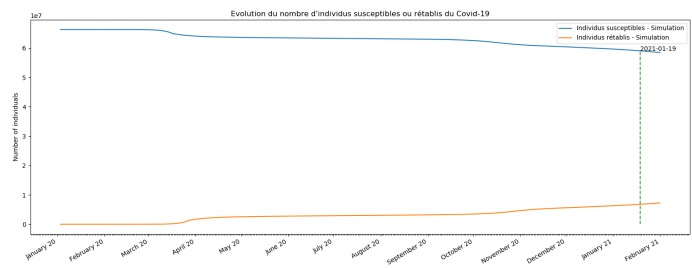

(b) Example response for number of susceptible and recovered individuals.

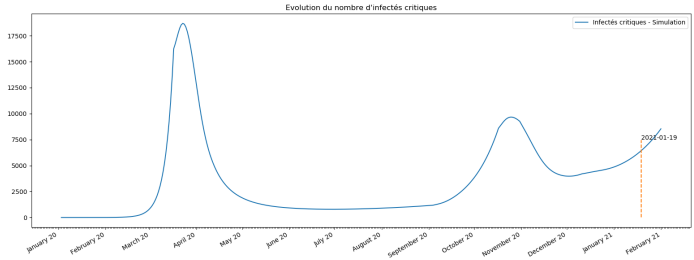

Figure 5: Example of a full simulation of the French epidemics. The official deaths and ICU numbers on Figures (e) and (f) are collected from Santé Publique France open data available on January 19, 2021.

\section{Limitations and Confrontation with Observed Statistics}

We now underline the following limitations which have been identified for this model:

- Fixed length for compartment $J$ - This length is determined by the generation time distribution $\zeta$. The restriction comes from the fact that we only consider here the average contagion period of individuals.

- Deaths not accounted by this model - According to the transitions admissible in this model, people outside hospitals cannot die: deaths in nursing homes or at home are not included. According to INSERM data, 1,362 covid-related deaths at home were recorded over March and April in France. An estimated 10,560 people died in nursing homes (EHPAD or EMS) in 
France by September 22, 2020. For the case of France, this represents about half of the total number of deaths which is an overwhelming fraction of the lethal impact of the virus. However the impact on this part of the population should probably be better described by dedicated models.

- Constraints on the temporal subdivisions of states - Relaxing these constraints would better capture the actual characteristics of the propagation but at the same time, would significantly increase the model complexity and the uncertainty on the initialization of the simulation as well.

- Relevance of compartment $W$ - The choice of considering compartments $H$ and $D$ can be supported by the fact that their corresponding statistics are collected in hospitals. In this respect, the compartment $W$ may seem artificial since it combines two completely different situations: (i) either it reflects diverse choices of treatment and care for patients in critical condition, or (ii) it covers the case where the ICU is overloaded. A finer model may be proposed to handle this particular category.

\section{Next Steps}

Working on a transparent and reproducible version of the model was the first major motivation of the present work. It offers a building block for the policy and decision maker toolbox. In order to enrich this toolbox, future work based on the current approach may focus on a sensitivity analysis on the parameters using Sobol indices for instance. It would allow policy makers to make a better use of the model outputs depending on which data are available at a precise point in time during the epidemic. The sensitivity analysis can also drive the effort for the collection of data supporting the estimation on input parameters. Indeed, in order to lower the dispersion of the simulation output, the precision on highly sensitive input parameters should be improved. Future work may also focus on how to specify a method for initializing the model based on publicly available data. This initialization consists of determining the number of individuals in each compartment and sub-compartment at a chosen start date. For instance, a warm start approach taking advantage of known statistics for other countries/regions or for similar epidemics may be developed.

\section{References}

[1] L. Bao, W. Deng, H. Gao, C. Xiao, J. Liu, J. Xue, Q. Lv, J. Liu, P. Yu, Y. Xu, F. Qi, Y. Qu, F. Li, Z. Xiang, H. Yu, S. Gong, M. LiU, G. Wang, S. Wang, Z. Song, Y. Liu, W. Zhao, Y. Han, L. Zhao, X. LiU, Q. Wei, and C. Qin, Lack of Reinfection in Rhesus Macaques Infected with SARS-CoV-2, bioRxiv, (2020). https://doi.org/10.1101/ 2020.03 .13 .990226$.

[2] G. Chêne, J-C. Desenclos, S. Vaux, C. Campese, and Fa. Ait El Belghiti, COVID-19 Point épidémiologique hebdomadaire du 4 juin 2020, 2020. Santé Publique, France.

[3] L. Di Domenico, G. Pullano, C.E. Sabbatini, P-Y. Bö̈lle, and V. Colizza, Impact of lockdown on COVID-19 epidemic in Ile-de-France and possible exit strategies, medRxiv, (2020). https://doi.org/10.1101/2020.04.13.20063933.

[4] R. Duidjou-Demasse, Y. Michalakis, M. Choisy, M.T. Sofonea, and S. Alizon, Optimal COVID-19 epidemic control until vaccine deployment, medRxiv, (2020). https://doi.org/ 10.1101/2020.04.02.20049189. 
[5] H. Salje, C. Tran Kiem, N. Lefrancq, N. Courtejoie, P. Bosetti, J. Paireau, A. Andronico, N. Hozé, J. Richet, C-L. Dubost, Y. Le Strat, J. Lessler, D. LevyBruhl, A. Fontanet, L. Opatowski, P-Y. Boelle, and S. Cauchemez, Estimating the burden of SARS-CoV-2 in France, Science, (2020). https://doi.org/10.1126/science. abc3517.

[6] M.T. Sofonea, B. Reyné, B. Elie, R. Djidjou-Demasse, Ch. Selinger, Y. MichaLAKIS, AND S. ALIZON, Epidemiological monitoring and control perspectives: application of a parsimonious modelling framework to the COVID-19 dynamics in France. working paper or preprint, May 2020.

[7] R. Verity, L.C. Okell, I. Dorigatti, P. Winskill, C. Whittaker, N. Imai, G. Cuomo-Dannenburg, H. Thompson, P.G.T. Walker, H. Fu, A. Dighe, J.T. Griffin, M. Baguelin, S. Bhatia, A. Boonyasiri, A. Cori, Z. Cucunubá, R. FitzJohn, K. Gaythorpe, W. Green, A. Hamlet, W. Hinsley, D. Laydon, G. Nedjati-Gilani, S. Riley, S. van Elsland, E. Volz, H. Wang, Y. Wang, X. Xi, C.A. Donnelly, A.C. Ghani, And N.M. Ferguson, Estimates of the severity of coronavirus disease 2019: a model-based analysis, The Lancet Infectious Diseases, (2020). https://doi.org/10.1016/ S1473-3099 (20)30243-7.

[8] F. Wu, A. Wang, M. Liu, Q. Wang, J. Chen, S. Xia, Y. Ling, Y. Zhang, J. Xun, L. Lu, S. Jiang, H. Lu, Y. Wen, And J. HuAng, Neutralizing antibody responses to SARSCoV-2 in a COVID-19 recovered patient cohort and their implications, medRxiv, (2020). https: //doi.org/10.1101/2020.03.30.20047365. 